\title{
Degenerative changes in Lumbar spine on MRI: A retrospective study
}

\author{
Jain $\mathbf{M}^{1}$, Vijaywargiya $\mathbf{M}^{2}$ \\ ${ }^{1}$ Dr Megha Jain, Department of Radiodiagnosis, L. N. Medical College, Bhopal, ${ }^{2}$ Dr Manisha Vijaywargiya, Department \\ of Anatomy, L. N. Medical College, Bhopal, MP, India.
}

Address for Correspondence: Dr Megha Jain, Associate Professor Department of Radiology, L. N. Medical College, Bhopal. Email- meghabc@yahoo.com

\begin{abstract}
Introduction: The widespread prevalence of patients complaining of backache has resulted in Spine as the most frequently requested neuroimaging examinations. Various imaging modalities like X-ray, myelography, discography and CT scan have been used to study the cause of backache in the past. However with the advent of Magnetic Resonance Imaging (MRI), revolutionary changes have occurred in the study and management of degenerative spinal diseases due to its superior soft tissue contrast resolution and its ability to define the anatomy, underlying pathophysiology and biomechanics of degenerative disc disease. Method: The present study is a MRI based retrospective study, conducted on 100 patients of lower backache segregated on the basis of age and level of lumbar spine involvement. Result: MRI findings suggested that maximum number of patients with complaint of lower backache refererred for MRI belonged to 40-60 years age group with most earliest and most frequent degenerative changes found to be in the intervertebral disc with maximum affection at L4-5 level followed by L5-S1 level. Conclusion: MRI has emerged as a non invasive multiplanar imaging modality with a superior soft tissue contrast resolution which can better define degenerative changes in the spine. In our study the earliest and most frequent degenerative changes were found to be in the intervertebral disc with maximum affection at L4-5 level.
\end{abstract}

Keywords: Backache, Lumbar spine, Disc, Degeneration, MRI.

\section{Introduction}

MRI is a non invasive multiplanar imaging modality which due to its superior soft tissue contrast resolution has the potential to define the anatomy, underlying pathophysiology and biomechanics of degenerative disc disease and is able to display the severity of neural compression and extent of canal stenosis. A variety of biochemical and structural changes take place in the spine during the process of aging and degeneration. Paula De Candido et al, in their MRI based study on distribution of degenerative changes in lumbar spine concluded that these degenerative changes increase with age and affect predominantly lower lumbar spine [1]. Backache may result from various causes; degenerative changes have been implicated as one of the leading cause of disability among adult population. In 60-80\% of all working adults suffer from backache at some point in their lives and almost all population over 60

Manuscript received $10^{\text {th }}$ May 2016

Reviewed: $24^{\text {th }}$ May 2016

Author Corrected: $4^{\text {th }}$ June 2016

Accepted for Publication $18^{\text {th }}$ June 2016 years of age show some degenerative changes in the spine, as a sequelae of physiological aging process or anatomical variation or secondary to previous trauma / pathology which hastens the degenerative process.

\section{Imaging Consideration}

Degenerative spinal changes can be of :

1. Bony origin : Osteophytosis, facetal hypertrophy, spondylolisthesis and spondylolysis.

2. Discogenic origin : Disc dessication, bulge, protrusion, prolapse / herniation.

3. Ligamentous origin : hypertrophy/thickening of spinal ligaments esp. ligamentum flava and posterior longitudinal ligament

Todd et al studied role of MRI in imaging of lumbar degenerative disc disease and its clinical and surgical relevance [2].

Based on MRI, the degenerative changes seen are as follows: 
1. Degenerative process begining in the intervertebral disc can be picked up at an early age of 20 years. Initially increase in water content of nucleus pulposus results in generalized bulge and focal herniation through the cartilaginous end plates of adjacent vertebra known as Schmorl's node.

2. Simultaneously marrow signal changes occur in the vertebra adjacent to the intervertebral disc.

Modic et al observed and described three types of vertebral marrow changes $[3,4,5]$ :

Type I changes appear as decreased signal intensity on T1WI and increased signal intensity on T2WI -this pattern represents bone marrow edema.

Type II changes consist of increased signal intensity on T1WI and are slightly hyperintense in appearance on T2WI due to fatty marrow changes as a result of marrow ischaemia.

Type III changes correspond to subchondral bone sclerosis and exhibit decreased signal intensity on both $\mathrm{T} 1$ and $\mathrm{T} 2$.

1. With time, the nucleus pulposus undergoes dehydration resulting in loss of height and intervertebral disc space- the disc becomes brittle, fibrous and loses elasticity- also known as 'Disc Dessication'- this is seen as progressive decrease in bright signal intensity of central nucleus pulposus of the disc due to loss of water content on T2WI and STIR. Pfirmann et al introduced MR classification of intervertebral disc degeneration, dividing them into five grades as per height and signal intensity [6].

Later, Griffith et al modified the scale into eight grades, by further scaling as per percentage reduction in the height of the disc [7].

2. As degeneration progresses, small circumferential fissures develop in the annulus fibrosus seen as linear areas of high signal intensity within the disc representing fluid filled crevices in a dessicated fissured disc- three types of tears were described by $\mathbf{Y u}$ et al namely concentric (Type I), radial (Type II) and transverse ( Type III). He concluded that disc bulge of greater than $2.5 \mathrm{~mm}$ are associated with annular tear [8].

3. Chronic sequelae of disc degeneration is herniation, stenosis and instability.
4. With increased mechanical stress the disc degeneration may progress into herniation which can be better defined on MRI by differentiating disc bulge from protrusion, extrusion, migration and sequestration depending on partial or complete annular tear and attachment or detachment from parent disc [9].

Disc bulge - smooth circumferential extension of the disc margin beyond the boundary of adjacent vertebral end plates with intact but weakened annulus fibres.

Disc protrusion- focal incomplete extension of nucleus pulposus through an incomplete tear of the annulus fibrosus limited by a persisting broad zone of attachment to the parent disc and an intact posterior longitudinal ligament.

Disc Extrusion- Extrusion of disc material through complete tear of annulus and posterior longitudinal ligament with a narrowed attachment to the parent disc

Sequestrated Disc- 'free fragment 'is when the extruded disc material detaches from the parent disc and migrates superiorly, inferiorly, laterally or posteriorly

1. Long standing degeneration of the disc give rise to bony overgrowth in adjoining vertebral margin- this marginal osteophytosis is seen as low signal protruberances at the paradiscal surface of vertebral body.

2. Once the discovertebral unit is disrupted, there are secondary effect on adjacent structures likefacet joint arthropathy which appears as irregular hypertrophy of the joint $+/-$ hyperintensity along the joint line and hypertrophy of ligamentum flava which appear as hypointense epidural areas of undue thickening along lamina and spondylolisthesis i.e. mal-alignment of the vertebral column.

3. Canal stenosis i.e narrowing of the spinal canal significant enough to cause neural compression, is multifactorial, with more than one of the above mentioned changes contributing to it.

4. Cord and the nerve roots appears as homogenously hypointense tubular structure which on compression may show altered signal intensity suggestive of myelopathic changes. 


\section{Material and Methods}

This study was carried out in the Department of Radiology L. N. Medical College \& J. K. Hospital and Research centre, Bhopal (M.P) India. It is a retrospective study. A sample of 100 patients in the age group of 20-80 years who presented with complaints of lower backache and were referred for MRI scan of lumbosacral region to the MRI department, in the year 2015, were randomly selected and included in this study. Patients with history of trauma or known spinal pathology or spinal anomaly were excluded from this study.

MRI was done on a Philips Multiva 1.5 Tesla Magnetic Resonance Imaging system (Koninklijke Philips N.V).

MRI Technique: Our aim was to obtain adequate information in the least amount of time. The total imaging time was approximately 12 minutes. The various pulse sequences (T1,T2, STIR) used in multiple planes (sagittal, axial and coronal) during examination of the spine have been shown to have a specific role.

T1 weighted sequence (short TR/TE) shows general anatomical details.

T2 weighted sequence (long TR/TE) is for tissues with high water content, edema, and demyelination.

STIR sequence is to evaluate marrow/soft tissue edema

\section{Imaging protocol :}

1. T1 TSE in Sagittal and axial plane (FOV 250 , slice thickness $3 \mathrm{~mm}$ )

2. T2 TSE in Sagittal and axial plane (FOV 250 , slice thickness $3 \mathrm{~mm}$ )

3. STIR in Sagittal and coronal plane

\section{Observation}

We found that maximum patients referred for MRI with low backache belonged to the age group of 41-60 yrs. The earliest change of degeneration appreciable on MRI observed in the intervertebral disc was in the form of disc dessication, seen as early as 20 yrs of age. Disc herniation and Lumbar canal stenosis were seen comparatively more in the age group of 61-80 yrs. Marrow changes and end plate defects showed variability. The degenerative changes were seen more commonly at L4-5 level in all age groups (Table-1). 74\% patients had L4-5 disc pathology, 52\% had L5-S1 while 28\% had L3-4 level involvement in our study.

Table-1: Showing age related distribution of level of spinal involvement with type of disc pathology and frequency of canal stenosis.

\begin{tabular}{|c|c|c|c|c|c|c|}
\hline \multirow[b]{2}{*}{ Age Gp. } & \multirow{2}{*}{$\begin{array}{c}\text { No. of } \\
\text { Patients }\end{array}$} & \multirow[t]{2}{*}{ Lumbar level } & \multicolumn{3}{|c|}{ Disc on MRI } & \multirow[t]{2}{*}{ LCS } \\
\hline & & & D & $\mathbf{D}+\mathbf{B}$ & $\mathbf{D}+\mathbf{B}+\mathbf{H}$ & \\
\hline $20-40 \mathrm{yrs}$ & 30 & $\begin{array}{c}\text { L4-5=L5-S1> L3-4 } \\
14=14>4\end{array}$ & 24 & 20 & 5 & 2 \\
\hline $41-60$ yrs & 40 & $\begin{array}{c}\text { L4-5 > L5-S1>L3-4 } \\
36>20>12\end{array}$ & 34 & 34 & 6 & 6 \\
\hline $61-80 \mathrm{yrs}$ & 30 & $\begin{array}{c}\mathrm{L} 4-5>\mathrm{L} 5-\mathrm{S} 1>\mathrm{L} 3-4 \\
24>18>12\end{array}$ & 30 & 16 & 8 & 14 \\
\hline
\end{tabular}

(D- desiccation, B- bulge, H- herniation, LCS- lumbar canal stenosis)

\section{Discussion}

The Spine is a collective term that comprises of:-

a) Vertebral column which encloses the spinal cord and is formed anteriorly by the vertebral body and intervertebral disc and posterolaterally by the neural arch.

b) The apophyseal joints, ligaments and soft tissue that support the vertebral column.

c) Neural tissue i.e. the spinal cord and its nerve roots.
The vertebral column has four curves- thoracic and sacral are convex dorsally, and cervical and lumbar are convex ventrally (appreciated in sagittal view). There are thirty three vertebra in human spine- seven cervical, twelve thoracic, five lumbar, five sacral and four in coccygeal with each having some unique characteristics. The shape of vertebral canal is- round at $\mathrm{C} 1$, triangular from $\mathrm{C} 2$ to $\mathrm{C} 7$, ovoid in thoracic and upper lumbar and again triangular in lower lumbar region (10). 
Disc Anatomy- The intervertebral disc interposed between two adjacent vertebral bodies alleviates shock and transmit forces applied to the spine. It is composed of three parts- the cartilaginous end plate covers the superior and inferior vertebral body surface, the annulus fibrosus is a peripheral collagen fibre ring forming a limiting capsule to the nucleus pulposus, and the centrally placed nucleus pulposus composed of well hydrated gelatinous matrix.

Thecal sac and epidural space- The thecal sac is formed by the meninges surrounding the spinal cord and its exiting nerve roots, which emerge from the spinal cord laterally and exit through the lateral recess / exiting neural foramina. The thecal sac is surrounded by epidural fat, venous plexus and neural elements which lie in the epidural space

Magnetic resonance imaging has proven to be an indispensable tool in clinical practice. Its value in assessing normal lumbar anatomy, internal disc chemistry and architecture, features of lumbar spine degeneration, and in diagnosing herniated lumbar discs has been well documented. Bradley K Weiner and Rikin patel in their study on fifty patients on MRI who were prospectively evaluated for disc containment on surgery, found MRI to be $72 \%$ sensitive, $68 \%$ specific, and $70 \%$ accurate in detecting containment status of lumbar herniated discs [11]. Degeneration can be considered as acceleration of the normal maturation process with age. The Degenerative process begins in the intervertebral disc as early as 20 years of age, and can be picked up earliest with the help of MRI. Sether et al described normal age related changes in MR signal intensity of the disc [12]. Minna O Erkintalo et al studied frequency, pattern and sequence of early degenerative changes in lumbar spine on MRI and concluded that degenerative changes emerged rapidly after the adolescent growth spurt and that they were more common in symptomatic adolescents at an earlier age [13]. Degenerative discal changes are predominant at L4-5 and L5-S1 level probably due to increased mechanical stress at the lumbosacral curvature. West et al in 2010 found that most frequently degenerated discs were seen at L4-5 31.2\% and L5-S1 30.6\%. In our study we found $74 \%$ affection at L4-5 and 52\% at L5-S1 [14]. Li-Peng Yu et al have also found that Modic changes in the vertebral marrow occurred most frequently at L4-5 and L5-S1, which is consistent with those reported by others [15]. Variation in spinal anatomy like transitional vertebrae and malalignment of the vertebra as in spondylolisthesis, is seen to hasten the process of degeneration seen in form of secondary changes like facetal arthropathy and ligamentum flava hypertrophy at adjacent level; Ken Hsu et al studied the incidence and etiology of high lumbar disc degeneration on MRI and concluded that isolated disc pathology was more common in age group of 24 to 48 years with mean of 40.7 years and was associated with pre-existing or coexisting abnormalities predisposing the disc to additional mechanical stress [16]. In our study of all the patients with low back pain, disc pathology was seen more in the age group of 40-60 years. Older age group however showed diffuse changes in lower lumbar spine with ascending pattern of higher lumbar disc involvement.

\section{Conclusion}

- Maximum Patients with clinically significant backache referred for MRI belonged to 40-60 years age group.

- The earliest sign of disc degeneration on MRI known as 'Disc Dessication' can be seen as early as 20 years of age.

- The degenerative changes are more common at L4-5 followed by L5-S1 level.

- Lumbar canal stenosis is more frequent in 61-80 years age group with L3 to S1 lumbar canal showing maximum affection.

Funding: Nil, Conflict of interest: None initiated. Permission from IRB: Yes

\section{References}

1. DeCandido P, Reinig JW, Dwyer AJ, Thompson KJ, Ducker TB. Magnetic resonance assessment of the istribution of lumbar spine disc degenerative changes. $\mathbf{J}$ Spinal Disord. 1988;1(1):9-15.

2. Emch TM, Modic MT. Imaging of lumbar degenerative disk disease: history and current state. Skeletal Radiol. 2011 Sep;40(9):1175-89. doi: 10. 1007/ s00256-011-1163-x. Epub 2011 Aug 17.

3. Modic MT, Steinberg PM, Ross JS, Masaryk TJ, Carter JR. Degenerative disk disease: assessment of changes in vertebral body marrow with MR imaging. Radiology. 1988 Jan;166(1 Pt 1):193-9.

4. Modic MT. Degenerative disc disease and back pain. Magn Reson Imaging Clin N Am. 1999 Aug;7(3):48191, viii. 
5. Modic MT, Ross JS. Lumbar degenerative disk disease. Radiology. 2007 Oct;245(1):43-61.

6. Pfirrmann CW, Metzdorf A, Zanetti M, Hodler J, Boos N. Magnetic resonance classification of lumbar intervertebral disc degeneration. Spine (Phila Pa 1976). 2001 Sep 1;26(17):1873-8.

7. Griffith JF, Wang YX, Antonio GE, Choi KC, Yu A, Ahuja AT, Leung PC. Modified Pfirrmann grading system for lumbar intervertebral disc degeneration. Spine (Phila Pa 1976). 2007 Nov 15;32(24):E708-12.

8. Yu SW, Sether LA, Ho PS, Wagner M, Haughton VM. Tears of the anulus fibrosus: correlation between MR and pathologic findings in cadavers. AJNR Am J Neuroradiol. 1988 Mar-Apr;9(2):367-70.

9. Osborn A.G.; Diagnostic Neuroradiology; Spine and spinal cord- Normal anatomy and congenital anomalies of the spine and spinal cord; I ${ }^{\text {st }}$ Edition; Elsevier; 1993; Chapter 19; Pages 785- 798

10. Byrne T.N., Benzel E.C.,Waxman S.G.; Disease of the Spine and the Spinal cord; Anatomy and Biomechanics of the spine and spinal cord; $1^{\text {st }}$ edition; Oxford University Press, Inc.; 2000; Chapter- 1; page3-16.
11. Weiner BK, Patel R. The accuracy of MRI in the detection of lumbar disc containment. J Orthop Surg Res. 2008 Oct 2;3:46. doi: 10.1186/1749-799X-3-46.

12. Sether LA, Yu S, Haughton VM, Fischer ME. Intervertebral disk: normal age-related changes in MR signal intensity. Radiology. 1990 Nov;177(2):385-8.

13. Erkintalo MO, Salminen JJ, Alanen AM, Paajanen HE, Kormano MJ. Development of degenerative changes in the lumbar intervertebral disk: results of a prospective MR imaging study in adolescents with and without low-back pain. Radiology. 1995 Aug; 196 (2):529-33.

14. West W, West KP, Younger EN, Cornwall D. Degenerative disc disease of the lumbar spine on MRI. West Indian Med J. 2010 Mar;59(2):192-5.

15.Yu LP, Qian WW, Yin GY, Ren YX, Hu ZY. MRI assessment of lumbar intervertebral disc degeneration with lumbar degenerative disease using the Pfirrmann grading systems. PLoS One. 2012;7(12):e48074. doi: 10.1371/journal.pone.0048074. Epub 2012 Dec 20.

16. Ken Hsu, James Zuchermann, Arthur White; High Lumbar disc degeneration: Incidence and aetiology; International society for study of the lumbar spine: May 1989.

\section{How to cite this article?}

Jain M, Vijaywargiya M. Degenerative changes in Lumbar spine on MRI: A retrospective study. Int J Med Res Rev 2016;4 (6):1030-1034.doi: 10.17511/ijmrr.2016.i06.28. 